\title{
Newquay (Cornouailles, Grande-Bretagne)
}

La plage dans la ville

\section{Christophe Guibert}

\section{OpenEdition}

\section{Journals}

Édition électronique

URL : http://journals.openedition.org/tourisme/148

DOI : $10.4000 /$ tourisme. 148

ISSN : 2492-7503

Éditeur

Éditions touristiques européennes

Édition imprimée

Date de publication : 1 juin 2014

Pagination : 80-92

ISSN : 2109-5671

Référence électronique

Christophe Guibert, «Newquay (Cornouailles, Grande-Bretagne) », Mondes du Tourisme [En ligne], 9 | 2014, mis en ligne le 30 septembre 2015, consulté le 14 novembre 2019. URL : http:// journals.openedition.org/tourisme/148; DOI : 10.4000/tourisme.148

\section{c) (i)}

Mondes du tourisme est mis à disposition selon les termes de la licence Creative Commons Attribution - Pas d'Utilisation Commerciale - Pas de Modification 4.0 International. 


\section{Newquay (Cornouailles, Grande-Bretagne) La plage dans la ville}

\section{Christophe Guibert}

Maître de conférences en sociologie

UFR ITBS, université d'Angers

Laboratoire ESO (UMR CNRS 6590)

[christophe.guibert@univ-angers.fr]

Résumé. La ville de Newquay, en Cornouailles (Grande-Bretagne) communique allègrement sur les ressources que constituent les plages et le surf aux fins d'identification territoriale. L'économie marchande est toutefois davantage l'affaire des entreprises privées ("surf shops", "surf lodges", etc.) que de la municipalité ou de l'office de tourisme local. II en résulte la multiplication de marqueurs de la plage et du surf dans la ville et hors les plages. Ce processus renforce le slogan affirmé de "beach lifestyle", mais brouille du même coup les limites spatiales et symboliques de la plage.

Abstract. Newquay, Cornwall (UK) communicates blithely on resources that are the beaches and surfing for territorial identification. However, the economy is more the case of private companies ("surf shops", "surf lodges", etc.) that of the municipality or the local tourist office. This leads to an increase of markers (beaches and surf) in the city and off the beaches. This process strengthens the "beach lifestyle" affirmed slogan, but blurs at the same time spatial and symbolic boundaries of the beach. 
D otée de près de vingt mille habitants, Newquay, une des principales "villes touristiques” (Stock, 200I, pp. I27-I3I) du littoral de la Cornouailles, a été, au début du XX $X^{\mathrm{e}}$ siècle, "mise en tourisme ${ }^{(1)}$ ". Elle est assez réduite sur le plan spatial, et les habitations sont concentrées autour d'un centre-ville comportant quelques rues piétonnes. Cette densité s'explique sans doute par le fait que la ville est située à l'extrémité d'une sorte de presqu'île où la côte est constituée de falaises. Newquay domine l'océan mais sans que les nombreuses plages, en contrebas de la ville, hormis Towan Beach à proximité du port, soient visibles : il n'est finalement pas possible, de la rue et du centre-ville, de voir les plages. Pour autant, celles-ci sont bien présentes, notamment par l'existence de marqueurs, de signes ou de référents disséminés dans la ville. Il n'est par conséquent pas nécessaire de voir la mer ou les plages à Newquay pour savoir que l'on se trouve dans une ville balnéaire à l'offre d'activités nautiques variée. Plus précisément, ce sont les "surf lodges", les "surf shops" et les écoles de surf qui, d'une part, marquent spatialement Newquay, indiquant sans le dire la présence des plages, et qui, d'autre part, rendent pérenne la mobilisation des plages (et des vagues) dans le temps puisque, moins qu'en France, la pratique du surf subit assez peu les effets de la saisonnalité touristique du lieu.

Si l'acception de la plage possédant des limites physiques est évidente, l'objet de cet article vise, à partir d'un cas singulier, à affirmer l'idée selon laquelle les limites spatiotemporelles et symboliques de la plage - cette dernière étant toutefois matérialisée par des signifiants bien réels empruntant au surf- sont quant à elles plus indécises. Ce constat liminaire alimente le principe d'hétérotopie qui caractérise la plage tel un "territoire singulier" (Rieucau et Lageiste, 2009, pp. 3-6 ; Coëffé, 2010 , pp. 5 I-68). "La plage dans la ville" - grâce au surf et aux "spots" de surf -, c'est à tout le moins l'idée que peut se faire le visiteur lorsqu'il habite touristiquement Newquay.

\section{Le cadre méthodologique ${ }^{(2)}$}

Les matériaux empiriques reposent sur une phase d'observation et d'une douzaine d'entretiens semidirectifs (auprès des gérants et moniteurs de surf, de la salariée de l'office de tourisme, de professionnels du tourisme) menés à Newquay, commune située en Cornouailles (Grande-Bretagne), en juillet 2013. Par observation, nous entendons la mobilisation de tous les sens du chercheur, "à commencer par la vue et l'oüe pour enregistrer les pratiques gestuelles et les discours" (Arborio et Fournier, 1999), mais aussi pour être attentif à la présence et à l'agencement des objets en lien avec la plage au sens large, ce social objectivé "qui s'est cristallisé dans des objets" (Pinçon et Pinçon-Charlot, 2002). L'utilisation de la photographie, eu égard à "sa force représentationnelle et analytique" (Piette, 2007, pp. 2328), est, dans ce texte, convoquée aux fins de description et de preuve.
Enfin, une analyse des documents de promotion du territoire des instances publiques en charge du tourisme puis des entreprises privées dans le secteur des sports nautiques a également été systématisée.

\section{LA VALORISATION DU SURF ET DES PLAGES PAR LES INSTANCES CHARGÉES DU TOURISME}

Le surf est, avec les plages, une des activités privilégiées dans la communication de la commune. "Newquay-Surf capital of the UK" ou encore "The adrenaline capital of the UK" sont les slogans de marketing territorial affirmés par la municipalité et le bureau chargé de la promotion touristique, le Tourist Information (ouvert toute l'année). Le site internet de l'office de tourisme (visitnewquay.org) est un relais utile pour valoriser et maintenir l'identification de la ville au surf. Jouant les cartes de l'ancienneté de la pratique, de la variabilité des spots de surf ou encore de l'offre de services disponible, les stratégies ne sont finalement pas très originales à l'échelle européenne - de nombreux territoires français, espagnols ou portugais mobilisent un tel argumentaire (Guibert 2006 ; 201 I, pp. 42-46) mais pourtant bien singulières pour l'Angleterre. Newquay est la seule ville anglaise à offrir une telle quantité d'espaces de pratiques et de services, tels les écoles et les magasins, et à pouvoir, comme Biarritz en France mais sans aller jusqu'à la "muséalisation" (Guibert, 20|4, 


\section{EnCAdRÉ 1 \\ DES COULOIRS DU MÉTRO LONDONIEN AUX CARTES POSTALES : NEWQUAY ET LE SURF SONT OMNIPRÉSENTS}

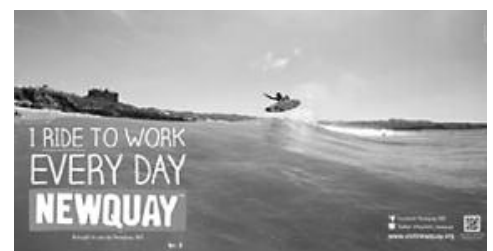

\section{Le métro Iondonien aux couleurs de Newquay}

Vingt-cinq stations du métro londonien ont affiché les slogans et images de Newquay en juillet et août 2013. Cette initiative émane du Newquay Business Improvement District (BID), dont le principal objectif est de valoriser l'image de la ville auprès de la clientèle touristique. Des publicités diffusées sur les ondes des radios FM anglaises renforcent cette attractivité avec le slogan suivant où la plage et le surf servent manifestement de support : "Orteils pleins de sable, peau salée et surf sensationnel".

\section{L'omniprésence du surf au sein des documentations à destination des touristes}

Seven : magazine gratuit comportant la liste des animations, des manifestations et des offres marchandes ; The Newquay Map : plan de la ville, disponible à l'aéroport et à l'office de tourisme.

\section{Fistral Beach : le haut lieu du surf anglais}

"International surfing centre", ainsi est qualifiée la plage de Fistral sur ce panneau disposé à proximité du parking de la plage et du golf de Newquay. Le décor est planté : c'est ici que le surf monopolise symboliquement et

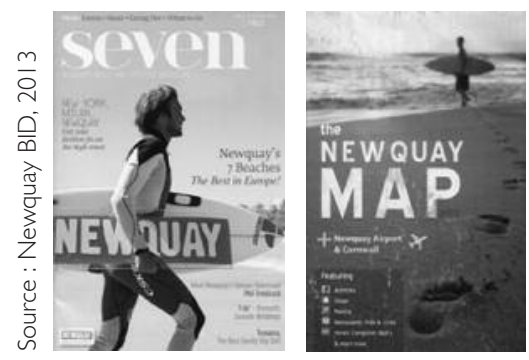
matériellement l'espace de la plage. $Y$ sont présentes de nombreuses écoles de surf privées et des surf shops, et y sont organisées des manifestations relatives au surf : un festival de musique (en partie sur la plage) avec des démonstrations de surf depuis 198I (le Boardmasters,
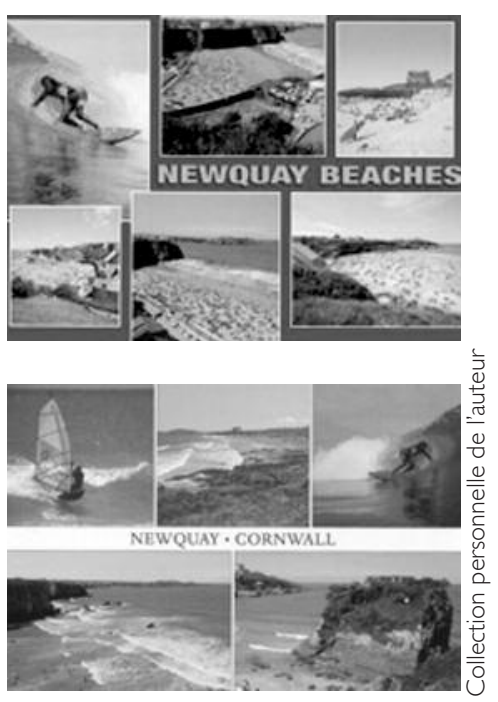
chaque mois d'août) et des compétitions professionnelles de surf depuis de nombreuses années. La commune de Newquay n'est pas partenaire économique de ces événements.

\section{Des cartes postales qui légitiment les pratiques et les lieux}

Partie intégrante de l'iconographie touristique, la carte postale met en image des caractéristiques et des singularités d'un lieu en mobilisant ce qui fait sens ("ce qui est emblématique d'un territoire(1)"). Les deux cas exposés ci-dessous contribuent à la communication de Newquay en mettant en exergue les plages et les usages sportifs de la plage (surf et planche à voile).

(I) Voir notamment le dossier "Cartes postales et tourisme" de la revue Espaces tourisme et loisirs, n 199, décembre 2002. 
pp. 187-193), mobiliser "l'authenticité" du surf ("le coeur historique") dans les discours :

"Il n'est pas exagéré de dire que Newquay est le cour historique du Royaume-Uni pour le surf depuis plus de 40 ans. Newquay est l'endroit idéal pour découvrir et apprendre le surf. [...] Newquay bénéficie de la meilleure gamme de magasins de surf, d'écoles et de fabricants de planches de tout le pays. Fistral Beach a la réputation d'être l'un des meilleurs "beach breaks' du pays. Fistral est capable de produire de puissantes vagues, creuses et tenant une bonne boule. Lusty Glaze, Towan, Great Western et Tolcarne sont des plages plus proches de la ville et fournissent également des vagues de surf de haute qualité. La chose la plus importante à Newquay est la culture du surf, $y$ compris au sein des écoles, très souvent gérées par des locaux experts qui aiment la plage, aiment le surf et aiment partager leur passion. Et vous serez entre de bonnes mains! À Newquay certains des meilleurs entraineurs et instructeurs $d u$ Royaume-Uni travaillent à vous faire profiter du 'fun' à chaque minute ${ }^{(3)}$ !"

Si l'identification territoriale de Newquay est bien effective, celle-ci tend à se renforcer ces dernières années. Des actions de promotion d'envergure nationale sont dorénavant mises au service du surf à Newquay. Au printemps 2013, le métro de Londres a ainsi été un lieu sélectionné pour promouvoir la destination : des affiches vantant la qualité des vagues et du surf à Newquay ont été disposées pendant plusieurs semaines. Les cartes postales, les panneaux signalétiques, les couvertures de magazines, etc. alimentent également le lien, devenu indiscuté, entre les plages, le surf et Newquay (cf. encadré 1$)$.

Les sept plages de Newquay sont également largement mobilisées en tant que "ressources territoriales" (Gumuchian et Pecqueur, 2004, p. 4). Chacune, plus ou moins proche du centre-ville, a sa propre histoire sociale et est valorisée comme telle dans les documents touristiques : plage familiale (Towan Beach), plage d'activités nautiques (Fistral Beach), plage familiale et populaire (Great Western Beach), plage avec des aménagements privés (Lusty Glaze Beach), etc. Le magazine Seven, dont le sous-titre est "Newquay's beach and lifestyle magazine”, n'hésite pas à affirmer que les sept plages constituent "the best in Europe" ! Véritables marqueurs territoriaux, ces plages et leurs spécificités alimentent les stratégies de marketing territorial des instances locales chargées du tourisme. La configuration spatiale de la ville, à l'extrémité d'une sorte de presqu'île, permet de choisir une plage en fonction du vent et de la houle soit pour s'en abriter, soit pour en profiter et pratiquer le surf, la planche à voile, etc. (cf. encadré 2$)$. Certains documents de promotion touristique comptabilisent jusqu'à douze plages différentes.

Le recours au surf et aux activités nautiques de plage à Newquay s'appuie, sans que cela soit affirmé dans les stratégies de marketing territorial, sur une longue histoire. En facilitant l'accès à la ville, la construction du chemin de fer dans les années 1950 a contribué à faire de Newquay, "the Cornish Riviera" (la "Riviera de la Cornouailles"), une destination touristique "à la mode" pour la clientèle anglaise en même temps que se développait la pratique du

\section{ENCADRÉ 2}

\section{UNE VILLE CEINTURÉE DE PLAGES}

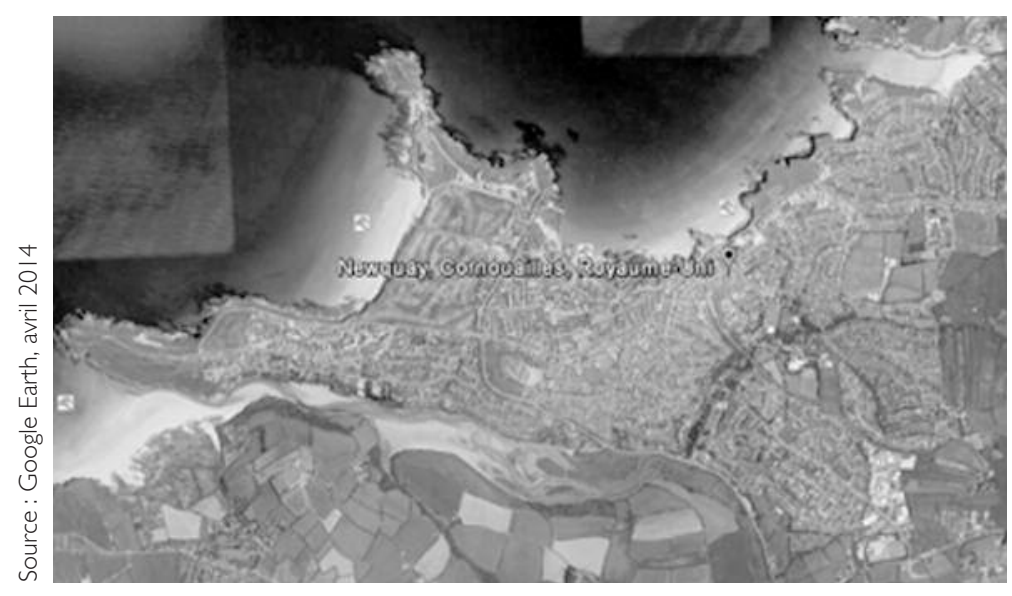


surf. Le surf y est présent dès les années 1940 (Mainsfield, 20 I I), soit une décennie avant la France. Lors de l'été 1959, la municipalité de Newquay emploie des sauveteurs australiens, dont certains sont des surfeurs, pour assurer la sécurité de la baignade sur les plages. Le "Newquay Surf Lifesaving Club" est créé par la municipalité, dans la foulée, sous l'impulsion d'un sauveteur sud-africain. Ce type de décision politique n'est pas nouveau ni isolé : de nombreuses communes littorales anglaises et des îles Anglo-Normandes emploient des Australiens (pour des raisons de proximité culturelle et historique) chaque été à partir du début

\section{Encadré 3}

LES “SURF LODGES” : UN HÉBERGEMENT TOURISTIQUE ALTERNATIF, À LA COMMUNICATION CIBLÉE
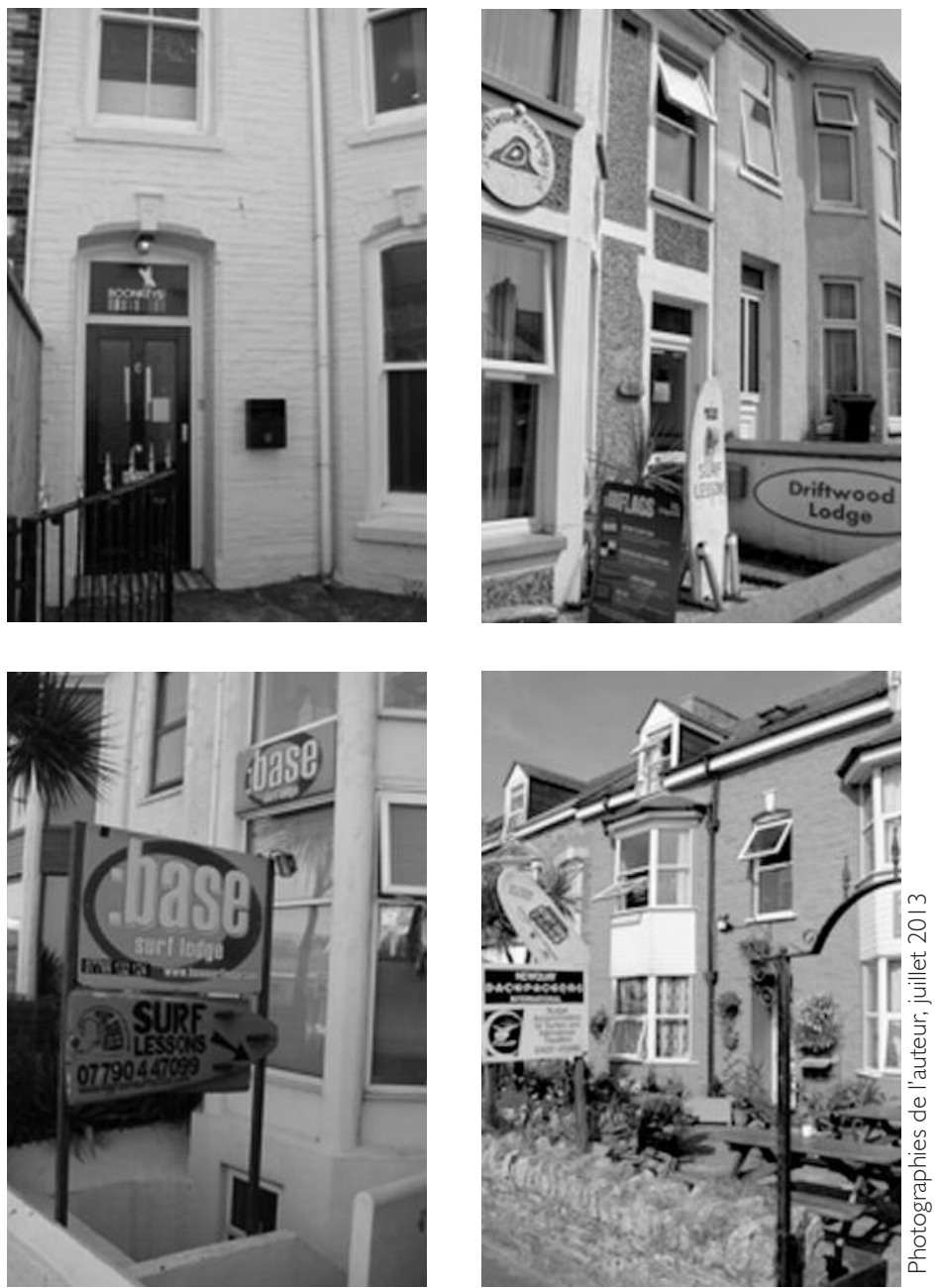
d'écoles de surf, leur fréquentation ou encore le nombre de surfeurs. Je ne sais pas s'il y un organisme qui s'occupe de cela ou si la ville de Newquay est intéressée. Je n'ai jamais eu connaissance de telles données chiffrées" (extrait d'un entretien dans les locaux du Tourist Information, Newquay, 16 juillet 2013).

Les plages à Newquay ne se singularisent pas uniquement à l'aune d'une communication touristique ciblée. Elles dépassent et franchissent en quelque sorte les limites qui leur sont physiquement attribuées (le trait de côte, les vagues, le sable, etc.) en pénétrant en ville.

\section{LA MATÉRIALISATION DU SURF ET DE LA PLAGE EN VILLE}

Si la pratique du surf est logiquement limitée à l'espace de la plage et des vagues, l'extension symbolique du domaine de la plage se construit à Newquay par un ensemble d'objets matériels et signifiants renvoyant directement au surf. Hors la plage et dans la ville, les objets symbolisant le surf notamment contribuent en quelque sorte à décloisonner la plage de son espace physique, questionnant en conséquence les limites (symboliques) de la plage.

\section{Les "surf lodges" : marqueur spatial et effet d'aubaine}

L'hébergement touristique à Newquay offre un type de prestations que l'on retrouve classiquement au sein des destinations où le surf figure parmi les principaux marqueurs du territoire concerné. Sont en effet dis- séminés à proximité des plages (un peu) ou dans le centre-ville (surtout) des "surf lodges", hébergements dont les tarifs sont assez limités en comparaison avec les hôtels 4 is et 5 光, nettement plus élitistes, de la commune. Ces hébergements consistent en un aménagement sommaire, voire spartiate pour la plupart : lits superposés, posters de surf sur les murs des chambres et télévision dans l'espace commun avec une collection de DVD sur le surf à disposition. La publicité est exclusivement orientée vers une clientèle de surfeurs : des planches de surf cassées et peintes, disposées près de l'accueil des établissements, matérialisent cette stratégie (cf. encadré 3). Les propriétaires de ces hébergements "spécialisés" peuvent, d'une part, être adossés à des écoles de surf, auquel cas l'école communique à la clientèle la possibilité d'être hébergé dans le lodgeet, inversement, les lodges communiquent sur les écoles de surf. Ils peuvent, d'autre part, être autonomes à l'égard des offres de services sportifs tout en mobilisant l'image du surf.

Si ce type d'hébergement contribue à moindres frais - eu égard à l'aménagement minimaliste des chambres et du service en général - à asseoir l'activité économique de Newquay en développant le nombre de lits touristiques, il renforce la présence du surf dans la ville, c'està-dire à distance des lieux de pratique. Les écriteaux, les panneaux et affiches permettant de localiser ces hôtels renforcent, comme les surf shops, l'idée selon laquelle le surf est omniprésent et incontour- nable à Newquay.

Ces établissements appartiennent à des propriétaires dont l'investissement dans l'identification de Newquay au surf est variable. Si quelques lodges sont explicitement en contrat avec des écoles de surf tel que le Driftwood lodge, par exemple, d'autres sont davantage autonomes à l'égard de l'économie directe du surf. John et Mary, propriétaires d'une grande maison dans le centre de Newquay, ont ainsi aménagé une partie de leur habitation en surf lodge avec une entrée distincte de la leur. Un tel choix permet à ce couple (John, 56 ans, est cadre moyen et Mary, 52 ans, sans emploi depuis trois ans) d'augmenter significativement ses revenus lors de la période estivale, constituant en quelque sorte une aubaine sur le plan économique : "On a décidé d'ouvrir un surf lodge il y a maintenant quatre ans. On avait remarqué que ce type de logement se multipliait ici à Newquay et que finalement certains arrivaient à se faire un vrai revenu supplémentaire. En plus on a des amis qui se sont lancés il y a déjà pas mal d'années et ça fonctionnait bien. En fait, maintenant que nos enfants ont quitté la maison et que par ailleurs, euh... j'ai eu des difficultés à trouver un emploi stable dans la région, eh bien on s'est dit avec mon mari que ça pouvait être une opportunité intéressante sans trop de risque. En plus mes fils font du surf, alors ça a été facile de retrouver quelques posters pour décorer les chambres!" (extrait d'un entretien avec Mary, Newquay, 17 juillet 2013). 


\section{EnCADRÉ 4}

\section{LE SURF EN VILLE}

e surf occupe physiquement et symboliquement l'espace du centre-ville de Newquay. Les façades des murs donnant sur la rue où l'on aperçoit les planches à vendre, l'architecture moderne des magasins des marques internationales (où les planches de surf ne sont finalement pas visibles) ou les surf shops plus simples où sont entreposées les planches à louer, etc. positionnent le surf comme élément central des formes et symboles dans la ville de Newquay (à l'instar d'autres villes de surf, telles Hossegor en France ou Peniche au Portugal).
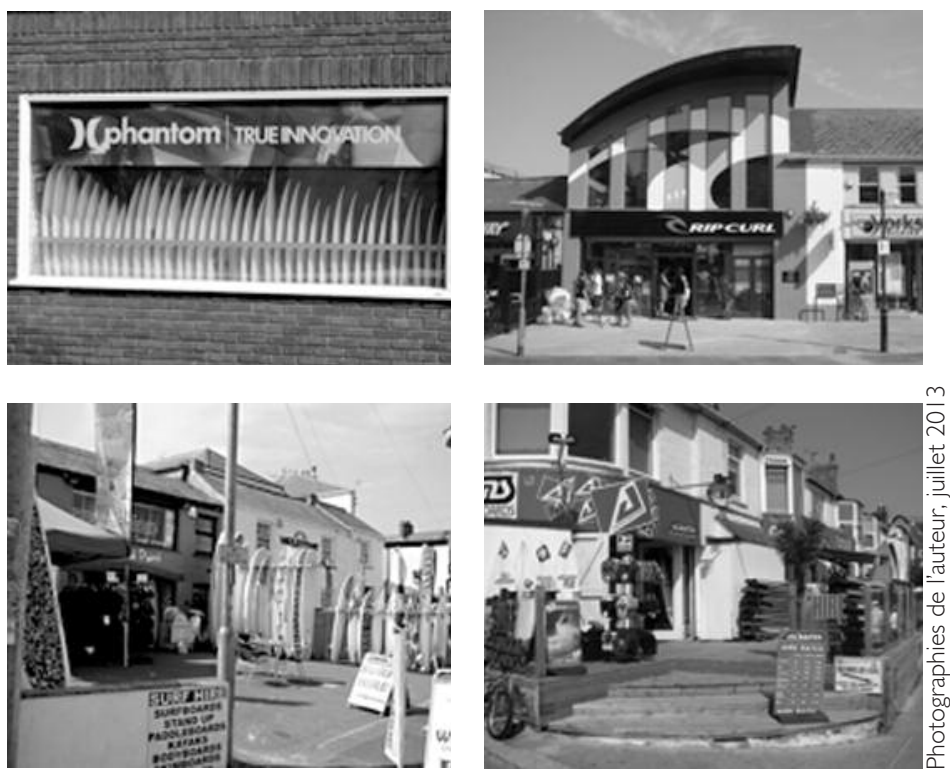

\section{L'omniprésence des surf shops}

La place du surf, à travers ses principaux signifiants $^{(5)}$ (la planche étant au centre de cet univers sémiotique), peut être analysée notamment à travers les points de vente et de location de matériel qui mettent en scène l'identité et les valeurs portées par l'enseigne. Ce sont principalement les écoles de surf (entreprises privées), les magasins spécialisés puis les surf lodges qui mobilisent cette image.

Les commerces dont l'activité est consubstantielle au surf, tels que les "surf shops" (vente ou location de matériels, prêt-à-porter ou objets plus ou moins dérivés de l'univers du surf), sont disséminés tout au long des principales rues - piétonnes ou non - du centre-ville de Newquay (cf. encadré 4). Il convient néanmoins de distinguer les petits commerces que sont souvent les surf shops des grandes enseignes mondialisées comme le magasin de la marque Quiksilver, par exemple, situé à Fistral Beach, ou celui de la marque Rip Curl, en centre-ville. Les premiers, parfois adossés à une école

\section{Encadré 5}

\section{JOUER LA CARTE DU SURF COÚTE QUE COÚTE ?}

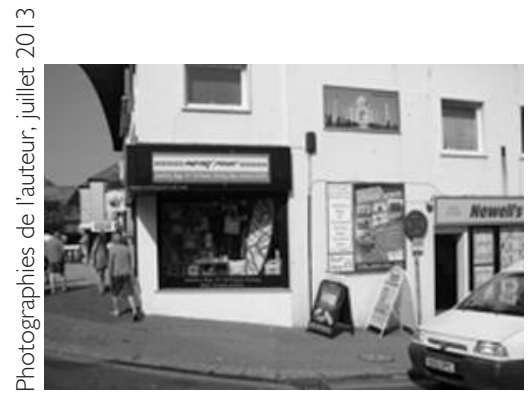

a vitrine du magasin Rocky Point, du nom d'un célèbre spot de surf d'Oahu, à Hawaii, et spécialisé dans la vente d'accessoires et de bijoux, expose de manière ostentatoire une planche de surf. Celle-ci, sans rapport avec les marchandises en vente dans le magasin, occupe néanmoins près du tiers de la surface visible de la vitrine. 
de surf, font souvent de la vente et la location de planches un enjeu économique important, à l'inverse des seconds où les vêtements et accessoires "à la mode surf” sont prédominants.

\section{Le surf comme outil}

\section{de communication}

Une autre configuration de commerces concerne les enseignes commerciales qui ne sont pas directement positionnées dans l'univers du surf mais qui mobilisent certains de ses signifiants. Ces enseignes ne peuvent être classées dans un secteur ou une gamme exclusifs : des magasins de prêt-à-porter, des bijouteries "bon marché", etc. utilisent les indices et marqueurs liés au surf. La planche de surf en particulier, visible et facilement intelligible, constitue un support de communication privilégié (cf. encadré 5). Newquay n'est toutefois pas le seul lieu touristique où l'image des activités nautiques est captée par des commerces n'ayant pas de rapport direct avec celles-ci(6).

Les plages et les usages de la plage apparaissent donc en ville. S'ajoutent aux signifiants et marqueurs précités des usages renforçant leur présence : des surfeurs traversent la ville en direction des plages les jours où les vagues sont présentes, planche sous le bras et revêtus d'une combinaison isotherme. Le codage culturel du lieu ${ }^{(7)}$ - la ville de Newquay, hors les plages - s'opère à la fois matériellement et symboliquement. Audelà de la présence en ville de marqueurs et de traces des plages et des activités nautiques telles que le surf, la plage et ses usages dépassent par ailleurs l'unique période estivale. La fréquentation des écoles de surf, singulièrement, permet en hiver de maintenir dans le temps une activité économique mais aussi la pratique des plages.

\section{LA PLAge TOUT AU LONG DE L'ANNÉE}

Il n'existe pas d'association sportive de surf à Newquay de type club comme l'on peut en trouver dans la majorité des communes littorales en France. Leur faible nombre (vingt en 2014) à l'échelle du pays renseigne d'ailleurs sur les pouvoirs relatifs de la Surfing Great Britain, la fédération anglaise de surf ("British Surfing Association" avant 2010), au sujet des prestations de services sportifs, notamment la découverte et l'apprentissage du surf. Si, en France, les associations agréées par la Fédération française de surf sont, à côté des entreprises (écoles commerciales), partie prenante de l'économie du surf en saison estivale, en Angleterre, et à Newquay en particulier, ce sont les entreprises qui, seules, proposent le surf comme activité sportive à la clientèle touristique. Les tarifs pratiqués (en moyenne 30 livres sterling la séance de deux heures en groupe ; 75 livres sterling la séance individuelle) témoignent d'un recrutement social relativement élevé chez les pratiquants souhaitant découvrir ce sport et s'y initier. Si l'offre des services visant à l'initiation au surf en été est très dense, elle n'en demeure pas moins présente hors saison touristique, et en particulier les mois de décembre à février. L'activité des écoles de surf contribue à maintenir le "beach lifestyle" (selon les termes du magazine Seven) tout au long de l'année.

\section{Une offre saturée en été ?}

La densité des écoles de surf en été est remarquable (cf. encadré 6). Si le problème de la régulation quantitative et spatiale de l'enseignement du surf se pose en France, dans quelques communes du sud des Landes et des Pyrénées-Atlantiques notamment, il est absent à Newquay. La ville compte entre vingt-cinq et trente entreprises ${ }^{(8)}$ d'initiation et de découverte du surf (soit davantage que les villes de surf françaises comme Biarritz ou Hossegor). Il en résulte une monopolisation de la plage par les moniteurs et les élèves.

Owen ${ }^{(9)}$, moniteur de l'école Ocean Crest, déplore le nombre trop élevé d'écoles de surf sur la commune. Il diversifie son activité économique en proposant une multitude d'activités nautiques en plus du surf : "Ici à Newquay, il y a trop d'écoles de surf, il y en a partout, sur toutes les plages! On est trop nombreux. Donc j'ai été obligé de me diversifier pour offrir différentes activités. On propose $d u$ surf rafting, $d u$ coasteering [saut de falaise dans la mer], $d u$ jet ski, $d u$ stand up paddle, $d u$ kayak, de la pêche, etc. Cet aprèsmidi par exemple, j'ai une réservation pour trente enfants en kayak de mer!"

Paul, gérant du Surfing School Activity Centre, confirme ce constat 


\section{ENCADRÉ 6}

\section{FISTRAL BEACH : "LA" PLAGE DE SURF DE NEWQUAY (VICTIME DE SON SUCCÈS)}

D lage la plus vaste de Newquay et la plus propice à la pratique du surf en raison de son orientation par rapport à la houle, Fistral Beach compte chaque matin, en été, une vingtaine de groupes de surfeurs inscrits à une leçon ou à un stage. Dès le parking, le décor est planté avec les multiples planches et combinaisons isothermes qu'il est possible de louer à la journée ou à la semaine. Chaque entreprise possède sa propre couleur de lycra, permettant aux membres du groupe de se repérer et au moniteur de concentrer les élèves.
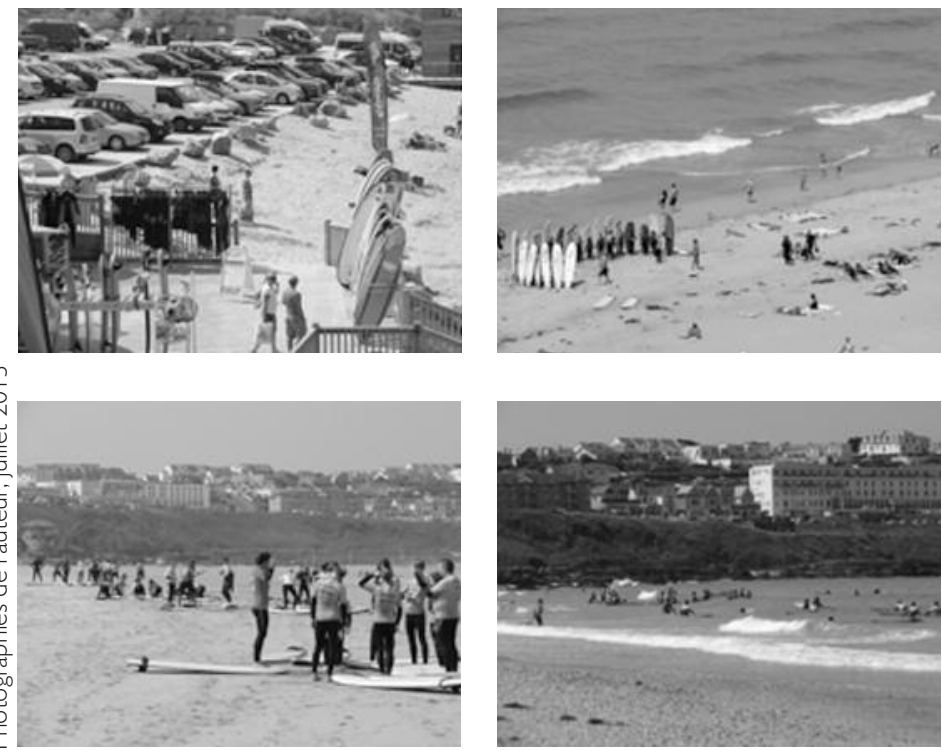

mais juge que la réputation de Newquay implique cette forte présence des entreprises commerciales comme les écoles et les magasins de surf : "Ici, il y a une vingtaine d'écoles et entre vingt et trente surf shops! C'est beaucoup. Mais Newquay est la ville du surf en Angleterre, elle communique sur cette image, c'est donc assez normal $q u ' i l y$ ait autant d'entreprises."

\section{Travailler l'été, voyager l'hiver}

Si une activité commerciale existe l'hiver à Newquay, la plupart des gérants déclarent toutefois fermer lorsque la fréquentation est trop faible. Les cas proposés ci-dessous témoignent d'une réalité somme toute assez ordinaire sur le littoral européen.

Paul, le gérant de la Surfing School Activity Centre, est également moniteur de surf. Il gère son entreprise six mois par an : "L'hiver, je pars six mois en Australie : donc six mois $i c i$ et six mois là-bas. Finalement, mes revenus me permettent de travailler six mois pour ensuite voyager le reste de l'année." Paul déplore cependant la précarité économique des moniteurs freelance, autrement dit sous statut d'indépendants: "Les moniteurs que j'emploie, l'biver, soit ils ne font rien, soit ils font d'autres petits boulots. Ils sont assez souvent employés en tant que serveurs dans les restaurants ou les bars: ils cumulent deux ou trois jobs à la fois. Mais ceux à qui suffit le salaire de moniteur de surf lors de la saison estivale, les plus paresseux, ils ne travaillent pas l'hiver. Mais je ne sais pas si c'est suffisant pour voyager à travers le monde l'hiver!"

Pour Owen, d'Ocean Crest, les conditions climatiques sont trop dures : l'école est ouverte d'avril à novembre. La période hivernale est mise à profit pour voyager ou réparer un lodge destiné à la location l'été : "Tous les deux ans, je pars l'hiver au Costa Rica ou à Panama en vacances et pour surfer. Ici, l'biver, il fait trop froid, il pleut, il y a des tempêtes! Je n'ai pas de clients. Six moniteurs, en plus de moi, sont salariés de l'école lors de la saison estivale. L'hiver, ils ne font rien! [rires] ou certains vont faire la saison de ski dans les Alpes, en France notamment. Moi, hors saison, j'ai un lodge que je répare, je fais des peintures pour y accueillir des clients lors de la saison d'été."

Mark, 34 ans, moniteur de surf, est gérant d'une autre école de surf : 
"Le local appartient à des amis et moi je m'occupe de la vente de matériel et de l'école de surf." Le local, situé dans la principale rue marchande de Newquay, est composé d'un seul espace, assez réduit, où sont entreposées ça et la les combinaisons isothermes et les planches servant pour les cours de surf et le matériel neuf (combinaisons et planches également). La saisonnalité touristique de Newquay structure l'activité commerciale de l'école de surf de Mark, dont le gros de l'activité a lieu en saison estivale, tandis que le magasin est ouvert à l'année : "Ici, c'est une petite école. Si le magasin est ouvert toute l'année, l'école de surf est principalement ouverte d'avril à septembre, avec un pic en juillet et août, comme en France. Mais si des groupes de clients souhaitent suivre des cours de surf en hiver, je les accueille, mais c'est vraiment très rare. $90 \%$ de nos prestations au sein de l'école de surf, c'est l'été. Parce qu'ici, l'hiver, il n'y a personne! Mais du coup, c'est mieux pour surfer : il y a moins de monde à l'eau !" Pour autant, Mark avoue ne pas chercher à développer son activité d'école de surf à l'année. "Moi, je ne suis pas comme Escape Surf School, par exemple, qui propose des cours tout au long de l'année. Nous, on est une petite école : j'essaie de faire assez d'argent en saison estivale pour pouvoir voyager et surfer l'hiver [rires] ! Je ne cherche pas à donner des cours en hiver: on commence l'activité à partir des vacances scolaires en avril."

Selon Jane ${ }^{(10)}$, salariée de la Fistral
Surf School (ouverte d'avril à octobre), les conditions du métier sont difficiles : salaires peu élevés, précarité professionnelle, etc. sont des éléments avec lesquels les moniteurs doivent finalement compter. "Les moniteurs de surf ne sont pas employés l'hiver car l'école ferme. La plupart travaillent dans d'autres secteurs. Certains font la saison de ski à la montagne en Europe, dans les Alpes notamment. Mais ceux qui sont avec leur famille, qui ont des enfants, c'est plus difficile de bouger pour un autre travail hors saison. Certains moniteurs sont diplômés $d u$ supérieur ou sont étudiants et d'autres non. C'est un mix, en fait. Je pense que ceux qui ont un diplôme d'université restent moins longtemps dans le métier que ceux qui n'ont pas de diplôme du supérieur. Ils peuvent plus facilement changer de métier après quelques saisons ici. La plupart des moniteurs sont âgés de vingt à trente ans environ. Après trente ans, ils arrêtent le plus souvent car c'est un métier difficile, même si c'est à la plage! Le salaire moyen d'un moniteur freelance - il est payé 30 livres la séance par l'école - est d'environ 1000 livres par mois, parfois plus, 1200 livres s'il fait plus de cours par jour, par exemple trois séances par jour. C'est variable donc. Mais ce n'est pas très élevé..."

La diversification de l'offre (des supports de pratique ou des lieux voués à l'enseignement du surf) est une stratégie permettant de limiter les effets de la saisonnalité touristique. Pour Sarah' ${ }^{(11)}$, si l'hiver est plutôt une période calme ("Les moniteurs ici n'ont pas trop d'activité en hiver : beaucoup partent surfer aux Maldives ou au Sri Lanka”), les voyages à l'étranger organisés par son entreprise permettent de maintenir une activité commerciale tout au long de l'année : “L'été, il $y$ a sept moniteurs de surf pour répondre à la demande de la clientèle mais les moniteurs ne sont plus que deux hors saison. Un des deux reste ici dans la structure l'hiver tandis que l'autre organise des surf trips à l'étranger. Nous proposons des voyages an Maroc, an Nicaragua, au Costa Rica et en Indonésie. Les clients peuvent choisir entre le surf et le bodyboard le support qui leur convient le mieux. C'est une activité qui fonctionne bien et nos clients ne sont pas uniquement des Anglais : on a des Australiens, des Européens, etc."

\section{Une saisonnalité touristique Iimitée}

Si les conditions de pratique du surf sont difficiles en hiver en Angleterre (température de l'eau inférieure à 10 degrés, tempêtes, taille des vagues, etc.), à tout le moins davantage que dans le sud de la France ou la péninsule ibérique, la saisonnalité de l'activité marchande est, paradoxalement, moins structurante que sur le littoral atlantique français. L'offre touristique du littoral atlantique répond, en France, à des contraintes temporelles fortes avec une nette dissociation entre les saisons en termes de fréquentation (Guibert, 2012, pp. 77-92). Les offres d'activités nautiques à vocation tou- 


\section{ENCADRÉ 7}

\section{UNE ÉCOLE DE SURF OUVERTE À L’ANNÉE}

$\mathbf{E}$ scape Surf School propose principalement des cours de surf aux débutants et ne vend que peu de matériel. Située dans le centre de Newquay, près du port et d'une plage pour les cours, l'entreprise propose des vestiaires séparés selon le sexe (avec des rideaux "pour les filles de culture indienne ou chinoise, qui sont plus timides", indique la secrétaire) et des douches chaudes, tandis que certaines écoles obligent les clients à revêtir une combinaison isotherme sur la plage directement. Figure ainsi en bonne position à l'accueil la distinction "Certificate of Excellence" 5 次, décernée par Tripadvisor en 2013.
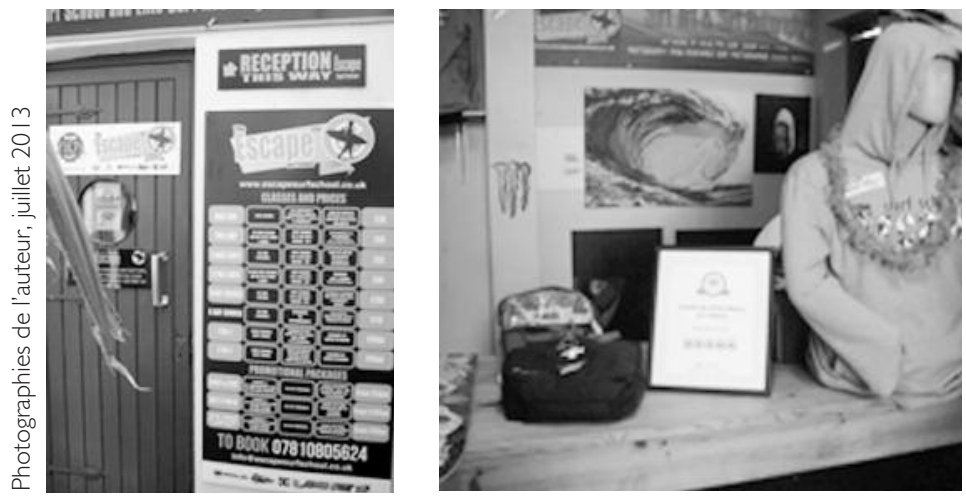

ristique, plus particulièrement, sont en conséquence étroitement liées à ces singularités littorales : aucune école de surf ne dispense d'enseignement du surf de type "initiation" ou "découverte" en hiver ${ }^{(12)}$. Seuls les clubs ont en réalité une activité hivernale, mais celle-ci est strictement réduite à l'entraînement des surfeurs aux pratiques compétitives. À Newquay, l'entreprise Escape Surf School est une des deux de la ville à être ouverte à l'année ( $c f$. encadré 7). Les caractéristiques matérielles de cette école singularisent les prestations : vestiaires, eau chaude, com- binaisons isothermes ou encore localisation dans le centre-ville sont autant d'atouts selon Mike ${ }^{(13)}$, le gérant, permettant de pérenniser l'activité commerciale hors saison : "Il y a deux écoles ouvertes toute l'année ici, ce sont les plus grosses, les autres sont fermées en hiver. Nous sommes cinq à six moniteurs en été et une secrétaire. L'biver, quatre moniteurs, qui sont en freelance chez nous, partent surfer vers d'autres mers... Le chiffre d'affaires est à $80 \%$ réalisé en été. Par exemple, on a en ce moment deux groupes par demi-journée : cet après- midi, une vingtaine de clients prendront des cours. Le week-end, c'est plein! Mais l'automne, ça marche bien aussi! En hiver, on a quelques clients individuels en semaine et des groupes le week-end. Les clients n'ont pas peur de l'eau froide: on propose des équipements complets pour rester an chand: combinaisons, gants, cagoules, chaussons."

La baisse de fréquentation en hiver ne signifie pas pour autant une diminution de la rentabilité. La baisse du nombre de moniteurs mais également les ressources économiques élevées de la clientèle permettent de maintenir l'activité de l'entreprise. Les clients en hiver "ont de l'argent" selon Mike. L'ouverture d'une ligne aérienne régulière (quotidienne) toute l'année entre Londres et Newquay explique également ce constat. "Les clients l'été sont originaires des environs ou de Londres, on a aussi des Allemands. En hiver, les week-ends, ce sont surtout de jeunes actifs de Londres qui viennent ici prendre des cours. Ils ont de l'argent! Ils s'initient au surf, vont à l'hôtel, au restaurant... ça permet de maintenir une activité."

Les propos d'Owen rejoignent ceux de Mike quant aux principales origines géographiques des clients de son école, l'Angleterre et les pays d'Europe du Nord: "Les clients sont issus de la région, de Londres ou Manchester et de l'étranger comme les Pays-Bas et l'Allemagne." L'habitude de pratiquer les activités nautiques dans une eau dont la température n'excède pas 16 degrés celsius en été et descend rarement en dessous de 10 degrés en hiver ${ }^{(14)}$ 
explique que, finalement, les touristes anglais et d'Europe du Nord ne voient pas dans la saison hivernale des conditions empêchant de pratiquer le surf. Les matériels proposés à la location par les écoles de surf répondent d'ailleurs aux exigences des clients (combinaisons isothermes suffisamment épaisses, chaussons en néoprène, etc.).

\section{ConcLusion}

Les plages, les spots et le surf sont, symboliquement et physiquement, attachés à la ville "touristifiée" de Newquay. Véritables ressources territoriales pour la municipalité et le BID (business improvement district), c'est toutefois davantage au sein de la sphère marchande privée que se structure concrètement l'utilisation du surf et des plages aux fins de commercialisation. L'analyse spatialisée de la ville et des signifiants présents, et les usages professionnels et touristiques propres au surf contribuent à faire de Newquay une ville de surf où le "beach lifestyle" est érigé en slogan performatif. Si la plupart des stations touristiques et villes touristiques balnéaires communiquent classiquement sur la ou les plages, force est de constater que celles-ci constituent un argument de choix, sinon le principal, dans la communication des instances chargées de la promotion de Newquay.

Cette spécialisation autour du balnéaire et des activités connexes n'a toutefois pas d'effet conséquent sur la structure sociale de la fréquentation touristique à Newquay : un public n'en chasse pas l'autre. La "trajectoire du lieu" (Équipe Mit, 20I I) est dans ce cas finalement assez homogène. Les hôtels et restaurants luxueux, le golf (construit en 1890), les villas opulentes en bord de mer se confondent avec l'architecture classique de la ville et maintiennent une image de station socialement élitiste. Eu égard aux tarifs pratiqués par les écoles de surf ou encore les prix du prêt-à-porter “à la mode surf”, la stratégie de communication des instances chargées du tourisme contribue ainsi, selon le principe de la reproduction sociale, à maintenir un recrutement social homogène dans le temps. L'histoire sociale de Newquay (culture locale, usages de la société locale, "ambiance" du lieu, recrutement social, etc.) permettrait de mesurer en quoi "l'effet de lieu" (Bourdieu, 1993; Ripoll et Tissot, 2010, pp. 5-7), c'est-à-dire le lien historique de Newquay à la plage et aux activités nautiques mais aussi les réalités matérielles et immatérielles, structure les rapports sociaux.

\section{Notes}

(I) Voir la synthèse des distinctions entre "site", "comptoir", "station" et "ville" touristique dans les travaux de l'équipe MIT en 20 I I. Port de pêche réputé, à la sardine principalement, depuis plusieurs siècles (mais aussi du commerce de plomb au XIX siècle en particulier) et comptant par ailleurs de nombreux agriculteurs dans sa population, Newquay est en effet une "ville touristique".

(2) Cet article est issu d'une recherche ("Emplois sportifs et saisonnalités touristiques : quels déterminants territoriaux ?') qui a été financée par le conseil scientifique de l'université d'Angers, 20 I I-2013.

(3) Source : site de l'office de tourisme de Newquay

[http://www.visitnewquay.org/activities/new quay-surf-capital-of-the-uk], consulté le 6 juillet 2013.

(4) Voir Guibert, 2006.

(5) Le terme renvoie à la théorie générale des signes de Peirce.

(6) Voir notamment Guibert et Coëffé

(2013, pp 89-96).

(7) Au sens de Jean-Didier Urbain (1994).

(8) II n'existe pas de recensement de ce type d'entreprises.

(9) Owen, 38 ans, est originaire de Newquay et a ouvert son école en 2006.

Le petit cabanon en bois, qui fait office de bureau et de lieu de stockage du matériel, est situé face au port de pêche de

Newquay.

(10) Jane, 25 ans est monitrice de surf et assure également les tâches d'accueil dans le local de l'école, situé à proximité du principal accès à la plage de Fistral beach, la plus renommée de Newquay pour la pratique du surf. C'est la seule école à avoir une structure "en dur" sur la plage, les 
autres écoles devant faire venir les planches et les clients en traversant la dune. Jane fait "depuis cette année toute la saison": "Les années précédentes, je n'étais présente que les mois d'été. En dehors de cette activité, je suis étudiante à l'université hors saison estivale."

(I I) Sarah, 27 ans, est salariée à l'accueil de l'école de surf Newquay Activity Centre. L'école est labellisée par l'English Surfing Federation et a un partenariat avec l'entreprise de matériel et de vêtements de surf Rip Curl.
(I2) Une enquête du comité départemen-

tal de tourisme des Landes de 2006 souligne que la fréquentation des clientèles des clubs et des entreprises offrant des services de découverte et d'apprentissage des activités nautiques s'élève à $70 \%$ de la fréquentation totale pour les mois de juillet et août, et à 93 \% de la fréquentation totale pour les mois de mai à octobre. (Source : CDT des Landes, Activités sportives et de loisirs, 2006, p. 18.)

(I3) Mike, 30 ans, est originaire "du Nord, des Midlands" : "Je suis arrivé ici à Newquay à l'âge de vingt ans, pour le surf, et, maintenant, le surf, c'est mon métier. J'ai monté cette école avec un associé." || est titulaire du "level 4", le plus haut niveau de formation délivré par la Surfing Great Britain.

( I4) L'écart de température en France est nettement plus accentué. Si la température peut descendre autour de 10 degrés en hiver sur la côte basque, elle monte régulièrement à 23-25 degrés les mois d'été, rendant sans doute moindre l'intérêt de surfer en hiver.

\section{Références bibliographiques}

Anne-Marie Arborio et Pierre Fournier, L'Enquête et ses

méthodes: l'observation directe, Nathan, 1999.

Pierre Bourdieu, "Effets de lieu", dans Pierre Bourdieu (dir.), La

Misère du monde, Seuil, 1993.

Vincent CoËFrÉ, "La plage, fabrique d'une touristi(cité) idéale", L'Information géographique, vol. $74, n^{\circ} 3,2010$.

Christophe GUIBERT, L'Univers du surf et stratégies politiques en Aquitaine, L'Harmattan, 2006.

Christophe GUIBERT, "La vague de Mundaka : une ressource territoriale convoitée", Jurisport, la revue juridique et économique du sport, $n^{\circ} 108$, avril 201।.

Christophe GUIBERT, "Les effets de la saisonnalité touristique sur l'emploi des moniteurs de sports nautiques dans le département des Landes", Norois, n²23, 2012.

Christophe GUIBERT, "Biarritz et le surf : une identification territoriale non figée", dans Olivier Bessy (dir.), L'Innovation dans l'événementiel sportif - De l'attractivité touristique au développement territorial, Presses universitaires du sport, 2014.

Christophe GUiBerT et Vincent CoËFFÉ, "Les enjeux touristiques de la marchandisation de l'image du surf à Oahu (Hawaii)", ESO

Travaux et Documents, $n^{\circ} 36$, décembre 2013.

\section{Hervé GuMUCHIAN et Bernard PECQUeUR,}

"La notion de ressource territoriale", Montagnes méditerranéennes, $n^{\circ} 20,2004$

Roger MAINSFieLD, The Surfing Tribe. A History of Surfing in Britain, Orca Publications, 201I (seconde édition).

ÉQUIPE MIT, Tourismes 3. La révolution durable, Belin, 201 I.

Albert PIETTE, "Fondements épistémologiques de la photographie", Ethnologie française, vol. 37, $\mathrm{n}^{\circ}$ I, 2007.

Michel PInçon et Monique PINÇON-ChARLOT, Voyage en grande bourgeoisie. Journal d'enquête, Puf, 2002.

Jean RieuCAU et Jérôme LAGEISTE, "La plage, un territoire singulier: entre hétérotopie et antimonde ", Géographie et cultures, n 67 , 2009.

Fabrice RIPOLL et Sylvie TISsOT S., "La dimension spatiale des ressources sociales", Regards sociologiques, n 40, 2010.

Mathis Sтоск, "Brighton and Hove : station touristique ou ville touristique ? Étude théorico-empirique", Géocarrefour, vol. 76, n² 2 ("Le tourisme et la ville"), $200 \mathrm{l}$.

Jean-Didier URBAIN, Sur la plage. Mœurs et coutumes balnéaires, Payot, 1994. 